\title{
From Scenarios to Use Cases, Test Cases and Validation Examples
}

\author{
K. Maki, A. Kulmala, K. Heussen, O. Gehrke, E. Rikos, J. Merino, M. Rossi, \\ L. Pellegrino, C. Sandroni, M. Z. Degefa, H. Taxt, D. Lagos, \\ and P. Kotsampopoulos
}

\section{Test Scenario Descriptions}

In the context of ERIGrid, scenarios are meant to be higher-level circumstance descriptions which will provide a basis for more detailed use case and test case definitions. As a term, scenario often refers to visionary descriptions of future development and the factors influencing it. Scenarios obviously apply long view perspectives where many uncertainties are present. In the context of ERIGrid, scenarios reaching to 2050 are of interest. In many cases, scenario work can feed in to political processes and decision making on different levels.

In the course of ERIGrid, generic system configurations have been considered more useful than traditional high-level scenarios. A system configuration approach allows including more detailed and quantitative data in the descriptions and providing a better technical basis for developing the use cases and test cases. Whereas high-level

K. Maki $(\varangle)$. A. Kulmala

VTT Technical Research Centre of Finland, Tampere, Finland

e-mail: kari.maki@vtt.fi

K. Heussen · O. Gehrke

Technical University of Denmark, Roskilde, Denmark

E. Rikos

Centre for Renewable Energy Sources and Saving, Athens, Greece

J. Merino

TECNALIA Research \& Innovation, Derio, Spain

M. Rossi · L. Pellegrino · C. Sandroni

RSE Ricerca Sistema Energetico, Milan, Italy

M. Z. Degefa · H. Taxt

SINTEF Energi AS, Trondheim, Norway

D. Lagos $\cdot$ P. Kotsampopoulos

National Technical University of Athens, Athens, Greece

(C) The Author(s) 2020

T. I. Strasser et al. (eds.), European Guide to Power System Testing, https://doi.org/10.1007/978-3-030-42274-5_6 
scenarios give some qualitative statements about the progress, system configuration uses quantitative data such as numbers of components, size of the system, etc. At the same time, the system configuration becomes more complex due to the amount of data but also more locally due to dimensions and local parameters.

The system configurations allow development of use cases, which give a description of a process leading to a specific objective. In other words, use case defines the actions needed to obtain some goal. Use cases are often described from an external perspective in a neutral manner, utilizing a formal methodology. Use cases can also be thought to define the interfaces of the process with its environment, inputs and eventual outputs.

Use cases can be defined from two perspectives: behavioural perspective and interaction perspective. Behavioural perspective is always function-type; it defines the behaviour of the process internally and towards external stakeholders. In the interaction perspective, most interest is on interactions between components and describing them, for instance by means of sequences.

Test cases with reference to system configurations require information on system parameters, ranges of parameters, system functionalities and quantitative measures. They also require information on test procedures and design of experiments. Test cases define the actual test setup; which are the combinations and series to be tested and which are the prevailing circumstances in which the tests are performed.

Following definitions have been used within ERIGrid [3]:

- System defined as a set of interrelated elements considered in a defined context as a whole and separated from their environment.

- System Configuration defined as an assembly of (sub-)systems, components, connections, domains, and attributes relevant to a particular test case.

- Scenario defined as a compilation of System Configuration, Use Cases, and Test Cases in a shared context.

- Use Case defined as a specification of a set of actions performed by a system, which yields an observable result that is, typically, of value for one or more actors or other stakeholders of the system.

- Test Case defined as a set of conditions under which a test can determine whether or how well a system, component or one of its aspects is working given its expected function.

\section{ERIGrid Generic System Configurations}

ERIGrid has defined three system configurations addressing key system areas [3]:

- Distribution grid

- Transmission grid and offshore wind

- Vertical integration 
The project has developed system configurations for these dedicated system areas as well as structures and templates for describing them. The templates apply similar hierarchy for the structure, starting from domain information and proceeding to more local information (such as area/level) and finally to individual components. Parameters are defined for each component as well as for the whole system as global parameters.

\section{Distribution Grid}

System configuration "Distribution grid" considers the electricity distribution system at MV and LV voltage levels. The area covered by this configuration starts at the HV/MV transformer, where also the responsibility area of DSO typically starts. On the low voltage side, the configuration is limited to the customer interface (metering point) or at the connection point of each active component or DER unit. However, the configuration also needs to consider components beyond the network connection point to the degree they impact on the state of the distribution grid. Hence components like control systems for DER units or controllable loads are included in the configuration.

The distribution grid as a domain includes a significant number of control-related challenges and developments. Communication is also increasingly present for monitoring and control purposes. One issue faced in this work was how to present these different layers. It could be possible to build up separate layers for the power system, communication system and control systems. This would enable a more detailed presentation of each system and especially of their interfaces. Eventually, control systems and ICT have been included as separate domains in this configuration. Multidomain components are located in domain interfaces, for instance smart meters which are physically connected to the power domain but also connected to the ICT domain in terms of data and control.

This system configuration includes a long list of traditional power system components such as lines, loads, transformers and switches. They all belong to the electrical power system domain. Some active components such as DER units, storage units, EV charging stations or intelligent controllers are also present; they are also physically connected to the electrical power system do-main, but they are also connected to control and ICT domains via their controllers and com-munications.

The system configuration also includes a heat system domain. The purpose of including a heat system is to be able to represent aspects of cross-impacts between heat and electricity; for instance, in a Combined Heat and Power (CHP) production, between heat exchangers, heat pumps, etc. However, heat system parametrisation is left very generic with the main focus to include connectivity.

The control domain includes various controllers connected with components. They have been categorized to central (coordinated) and local control methodologies. ICT domain includes metering systems, communication and data management areas. Stakeholders and markets have also been presented as separate domains, indicating different roles and markets within the scope of this system configuration. 


\section{Transmission Grid and Offshore Wind}

The offshore wind power plant scenario has been selected because it is a predominant future scenario with special operation characteristics and impact on transmission grids. For specifying the system configuration, the following assumptions have been made:

- A meshed HVDC network will be adopted because it seems a cost-effective solution for hosting high-power wind generation and, as a topic, it presents an additional research interest.

- AC grid parts are assumed for the connections of the wind power plants to the HVDC hubs and an aggregated representation for the on-shore substations/ connections.

- More than one connection to the shore may be used because it adds extra benefits in terms of services and allows the wind power plant to participate in various processes of operation and the energy market. Also, this increases the number of applicable use cases.

- Interconnection with different control areas (different countries) so as to increase diversity of operating characteristics and processes at the ends of the system.

- Simple configuration with the minimum possible number of components that at the same time satisfy the abovementioned requirements.

- Hierarchical control structure based on levels, with each level assigned with specific roles for the system's protection, operation and optimisation.

- The system is assumed to have specific role(s) in the energy and ancillary services market which help to establish concrete interconnections with the 'Market' domain.

- The interconnection with other physical domains such as weather conditions is more specific since there is only one RES technology involved. Nevertheless, the effects of weather conditions are considered only as a boundary of the system and are not analytically modelled.

Based on example scenarios, the system configuration is extended according to the aforementioned assumptions. To this end, components given in the basic scenario have been identified followed by components for possible extensions to the basic scenario. For those components, attributes and domains have been identified as well as the connections between.

One of the most crucial discussion topics was the importance of considering onshore wind power plants together with the offshore scenario. The former is (and will be) the predominant wind-production scenario of the future. However, taking into account only the share of a scenario for selecting it, it means that other largescale technologies should also be considered. Thus, only the offshore wind power plant scenario is considered, not just for its contribution to the RES share but also for its technical characteristics. Specifically, the incorporation of meshed HVDC grids is a value added for the selection of the scenario.

The topology of the system was also an important discussion topic. Among different options such as pure AC, radial DC, and meshed DC configurations, the meshed scenario has been selected which is technologically the most promising solution for bulk transmission of offshore wind power. 
HVDC onshore fault ride-through protection was also identified as a serious challenge from an operational standpoint, as well as from testing and simulation perspectives.

A third point of discussion was the way of modelling the onshore connection points and, in general, the overall onshore transmission grid's behaviour in combination with the selected scenario. To this end, aggregation of production/consumption at various grid nodes (at transmission level) and simplified representation of the transmission grid has been agreed. With the use cases in mind (e.g., fault ride-through, energy balancing, active power control, stability to a lesser extent) this is a plausible assumption.

\section{Vertical Integration}

The vertical integration scenario and system configuration provides a possible background for use cases requiring coordination and integration of transmission and distribution grid related tasks. In principle, it includes all domains used in other system configurations; however, in this system configuration often abstractions and aggregations of usually included components are employed, as the full detail may overload a given test requirement.

Due to its cross-cutting nature, vertical integration system configuration sets a lot of attention on connectivity of components, their information exchange as well as on the roles of stakeholders.

\section{Focal Use Cases}

The ERIGrid Focal Use Case Collection has been gathered during the project, based on existing outputs from earlier projects and networks. Several repositories, for instance EPRI (The Electric Power Research Institute) and SGCG (Smart Grid Coordination Group) ones have been utilized while building the ERIGrid collection.

Focal use cases have been categorised according to the service they provide for the system [4]:

- SS1 Energy balance

- SS2 Energy efficiency

- SS3 Power quality

- SS4 Power system stability

- SS5 Infrastructure integrity, protection and restoration

The following sub-sections present these services that can be provided at system level and show exemplary use cases for each of them. These use cases are aggregates of several use cases within the ERIGrid collection.

SS1 Energy Balance The energy balance of a network is a fundamental requirement for its operation; in fact, the generation has to constantly follow the demand curve in order to maintain the system stable. Taking into account the time horizon for which the ERIGrid system configurations have been developed, scenarios are 
included in which also the demand is controlled in order to match the generation availability. Here the energy balancing functions are defined as follows:

- Functions aimed at guaranteeing the long-term energy balancing and which have been categorized in SS1 Energy balance in the restoration of the planned power exchanges with external systems.

- Functions aimed at guaranteeing fast and prompt support in the restoration of the power balancing have been categorized as Focal UC in SS4 Power system stability.

The focal Use Cases listed below describe the selected functions for the support of system energy balance:

- SS1.SC1 Management of Flexible DERs for the Long-term Balancing (Frequency/Voltage Restoration Reserve) of Microgrids in Island-Mode

- SS1.SC2 Automatic Frequency Restoration Reserve from VSCs of Large Wind Farms

- SS1.SC3 Automatic Frequency Restoration Reserve from DERs

SS2 Energy Efficiency The containment of the losses in energy conversion, transportation and storage has always been one of the main objectives in the design and operation of power systems. In fact, several use cases can be found or deduced from literature specifically aimed at enhancing the energy efficiency of systems. Energy efficiency related use cases have high relevance among the ERIGrid collection:

- SS2.SC1 Optimal Distribution Network Control for the Reduction of System Energy Losses

- SS2.SC2 Optimal Transmission Network Management Level for System Energy Losses Reduction

- SS2.SC3 Incentivising Distribution Network Local Balancing to

- Minimize Transmission Network Loading

SS3 Power Quality The increasing penetration of distributed generation is particularly challenging from the power quality point of view and, currently, one of the most relevant limitations in terms of renewable integration are the voltage issues caused by generation at distribution level. In order to mitigate these effects, potential solutions have to be developed and most of them require the coordination of more resources in order to manage the voltage congestions. According to this, all the ERIGrid system configurations can be considered as proper scenarios in which power quality functions can be tested and, for each of them, specific focal use cases are listed:

- SS3.SC1 Advanced Voltage Control of Distribution Grids Supported by DERs Power Interfaces

- SS3.SC2 Voltage Quality Support by Onshore and Offshore (VSC-HVDC connected) Wind Power Plants

- SS3.SC3 Transmission Network Voltage Quality Support by the Distribution Network (VPP) 
SS4 Power System Stability Another particularly challenging aspect in future power systems is represented by stability. Most of the functions aimed at supporting the system robustness are currently performed by traditional generators. For scenarios in which their presence is expected to be less predominant, other solutions have to be exploited. In order to support the power system stability, many actors can be involved as well as all the domains considered within ERIGrid. Also in this case, on the basis of ERIGrid System Configurations, a list of use cases is presented:

- SS4.SC1 Management of Flexible DERs for the Instantaneous Active/Reactive Power Balancing of Microgrids in Island-Mode

- SS4.SC2 Large-scale Wind Power Plant (Onshore and Offshore VSC-HVDC Connected) Support in Frequency Containment Control and Power System Inertia

- SS4.SC3 DERs Support in Frequency Containment Control and Power System Inertia

SS5 Infrastructure Integrity, Protection, and Restoration Other functions that are expected to evolve in the ERIGrid system configurations are represented by the ones supporting the integrity, protection and restoration of the System Configurations' infrastructures. In fact, taking into account the high flexibility that energy players are able to provide at all power system levels, significant benefits can be provided through theses dedicated use cases. As for other services, use cases are listed, including also a use case describing functions aimed at guaranteeing ICT integrity, protection and restoration:

- SS5.SC1 Fault Detection and Corrective Management of Distribution Grid Assets and Energy Resources

- SS5.SC2 VSCs (of HVDC and Large Windfarms) Support During Transmission Network Restoration

- SS5.SC3 Intentional Islanding of Microgrids During Widespread Disturbances and Restoration of the Transmission System

- SS5.SC4 Identification of ICT Anomalies and Restoration of the Communication Links

These listed sixteen focal use cases have been considered representative of most relevant functions that can be reasonably expected to be operative in the ERIGrid system configurations. These focal use cases have been designed in order to cover a large spectrum of system domains and actors, and to comprehend several more specific functions. Based on this, several test cases can have been designed, taking advantage of the different domains which can be easily reproduced and/or simulated within ERIGrid research infrastructures.

\section{Test Cases}

ERIGrid covers all testing approaches consisting of virtual-based and/or real-worldbased methods. The test set-ups can be divided into four categories: (i) pure simula- 
tion (incl. co-simulation), (ii) Controller Hardware-in-the-Loop (CHIL) simulations, (iii) Hardware (HW) experiments, and (iv) Power Hardware-in-the-Loop (PHIL) experiments. These testing approaches can be considered to form a structured testing chain consisting of the following steps:

- Pure simulation: Virtual-based approaches both offline and in real-time. All aspects of the System under Test (SuT) are modelled using suitable software(s) and the accuracy of the results depends on the accuracy of the utilized models. Cosimulation can be used to combine different simulators that consider each domainspecific part of the SuT individually.

- CHIL experiments: Real control hardware is utilized in a closed-loop simulation of the system. Virtual-based and real-world-based approaches are combined. CHIL experiments enable more accurate simulations in case an exact model of the controller is not available. Communication delays, noise, execution time of algorithms etc. can be taken into account more easily than with a pure simulation approach. CHIL experiments can also be used to verify the correct operation of a specific control hardware.

- Hardware experiments: Open-loop testing of real components. This can be seen as the conventional part of component testing and the results are mainly related to component characteristics.

- PHIL experiments: Closed-loop testing of real components. Virtual-based and realworld-based approaches are combined. Interactions between the hardware under test and the overall system can be studied.

The next step after the four testing approaches would be demonstration in a real operational environment. The test cases are selected to cover all of the four testing approaches. Better RI integration is needed to enable comprehensive testing of multi-domain systems and also to enhance the already existing single-domain testing procedures. Better integration can be achieved by at least two means: By simplifying the process of porting an experiment from one RI to another, e.g., by using standardized interfaces, and by enabling joint use of RIs with different capabilities through a real-time communication between the RIs. Here the concept of an RI is understood to include off-line simulation tools as well as physical laboratory infrastructure consisting of real equipment such as generators and virtual equipment such as real-time simulators. Research questions determine the test objectives for each of the selected test cases. Additionally, previously defined system configurations and focal use cases as well as capabilities of different RIs are used as inputs for the selection process. The number of tested use cases is intentionally quite low so that the work can concentrate on the research questions on infrastructure integration. Same use cases are used for many different test cases so that the testing approaches can be developed and results of individual setups can be compared.

\section{Selected Test Cases}

The work concentrated on demonstrating and validating research infrastructure integration. Two types of test cases have been defined: In single-RI integration test cases, models, algorithms etc. developed in one RI are used in another RI as a part of a 
test but real-time communication between the RIs is not needed. Single-RI test cases are also used to compare different experiment set-ups to enable performing the same tests in different facilities. In multi-RIs integration test cases, the interfacing and real-time communication between the RIs is needed.

Technical challenges identified for single-RI test cases include comparison of different testing approaches and test setups, integrating third-party Software (SW) as a part of a test case and model transfer between RIs. Four single-RI test cases have been selected as presented below. The following test cases have been selected as single-RI test cases [2]:

- TC.S.1 Component testing at different RIs with different setup

- TC.S.2 Use of SW developed by RI1 in HW RI2

- TC.S.3 Use of component model developed in RI1 to perform multi-domain system tests in RI2

- TC.S.4 Test of distributed cyber-physical systems in RI1 as a monolithic setup in RI2

Technical challenges identified for multi-RI test cases include integration of remote software and integration of remote simulators or hardware. The multi-RI test cases are used both to validate the correct operation of interfaces developed and to demonstrate the real-time joint operation of research infrastructures for smart grid testing purposes. Two multi-RI test cases have been selected as presented below. The following test cases have been selected as multi-RI test cases [2]:

- TC.M.1 Integrate control SW running in RI1 with HW RI2

- TC.M.2 Extend HW resources of RI1 using resources of other RIs

More detailed implementation plans for each test case have been developed, started by constructing test specifications and experiment specifications as well as experiment setups. Full test case descriptions include system configurations as a basis and requirements for research infrastructures. System configurations provide information on the required components and connectivity.

\section{System Validation Examples}

The following sections present two more detailed examples utilizing the procedure. The first one deals with a voltage control application and the second one with the development of a converter controller. 


\subsection{Analysis of the Centralized Voltage Control for Rhodes Island}

This test case aims to demonstrate the "Hardware/Software integration between different Research Infrastructures". This test case aimed to demonstrate how modern advanced testing techniques, such as CHIL, can be used to fill the gap and ensure faster and more secure transition between pure simulations and field implementations. In this test case, a control hardware in the loop setup was used in ICCS RI in order to test a DSO's control algorithm of a Non-Interconnected Islanded Power System (NIIPS) in realistic conditions. A dynamic model of Rhodes Island system implemented in ICCS's real time digital simulator in full detail consisted of:

- Synchronous Generators and their control systems (automatic voltage regulators, governors, secondary frequency controls) of the 2 different power stations in the island.

- The HV network of the Rhodes Island system.

- The 5 controllable WTs that exist in Rhodes network (as average P, Q models).

- 5 average $\mathrm{P}, \mathrm{Q}$ models that represent the demand of the 5 different HV/MV substations of the island.

The dynamic model of the island power system was simulated in real time, sending also measurements and receiving setpoints from a controller hosting DSO's algorithm which operates in a CHIL setup as presented in the next figure. The first control algorithm measures the production of the synchronous generators (thermal units), the power produced by the WTs and the available power of the WTs. From those measurements the total demand is derived. The controller then determines the maximum production allowed cumulative by all the WTs according to their maximum available power, the maximum permitted penetration level that is set for stability purposes (e.g. 30\% of the total demand) and the non-violation of the minimum loading levels of generators. The algorithm then decides how to distribute this available power according to the nominal rating of each one of the 5 WTs as well as their respective available power at that moment. Finally, the setpoints for the thermal generators calculated by trying to reduce the production cost while at the same time supply the remaining demand and provide the required reserves for the safe operation of the system. Those setpoints sent alongside with the WTs setpoints back to the RTDS. Furthermore, this CHIL setup is ideal to examine the behaviour of the existing algorithm as well as a possible improvement of it in real time conditions (e.g. noise, time delays) and also in events that can cause stability issues in the system such us the largest WT disconnection. In order to illustrate this, an improvement of the DSO's centralized control tested also in ICCS CHIL testbed and compared to the existing controller. The proposed control algorithm also tries to reduce the voltage deviation from the nominal value, similar to the CVC, as well as to ensure stability due to RES penetration levels through a different approach. The controller hosting this algorithm receives measurements (WT Power, Thermal Unit Production, WT available power, active and reactive power demand of the $5 \mathrm{HV} / \mathrm{MV}$ substation) 
from RTDS, solves an optimization problem and sent back in RTDS the WT active and reactive power setpoints and the thermal generator setpoints. The optimization problem tries to minimize the voltage deviation and the operating costs according to the following objective function:

$$
\min _{x}\left\{w_{\text {cost }} \cdot \sum_{i=1}^{\infty} \operatorname{Cost}_{i} \cdot P_{g}+w_{v} \cdot \sum_{j=1}^{n}\left(V_{n}-V_{j}\right)^{2}\right\}
$$

Subject to the constraints:

- Power Balance equations in each node

- Voltage constraints $\left(V_{\min } \leq V_{j} \leq V_{\max }\right)$

- Angle Constraints $\left(-180^{\circ} \leq d_{j} \leq 180^{\circ}\right)$

- Thermal Generator Production Limits $\left(P_{\min } \leq P_{i} \leq P_{\max }\right)$

- WT power constraint according to the available power $\left(P_{i}^{W T} \leq P_{i}^{W T_{\text {avail }}}\right)$

- WT power factor constraint $\left(Q_{i}^{W T} \leq P_{i}^{W T} \cdot \tan \arccos 0.9\right)$

- Dynamic Frequency Constraints $\left(F\left(H, P_{d i s}\right) \leq 49.4\right)$

The last constraints are a set of linear constraints ensuring that the frequency will not drop below a frequency level (here $49.4 \mathrm{~Hz}$ which is the setting of the first acting Under Frequency Load Shedding (UFLS) relays and is a recorded transient that has caused black out in the Rhodes NIIPS). This is a different approach compared to the existing control which enforces a penetration limit to the WT power. Both methods try to ensure that if a contingency occurs (e.g. the largest WT is disconnected) the frequency remains within limits and no UFLS relays trip. The CHIL setup in ICCS infrastructures allows to run in the loop both algorithms and perform at the same time contingencies in RTDS in order to examine if the existing and the proposed control algorithm ensure safe operation. In addition, the CHIL allows both control algorithms to be tested in realistic conditions, ensuring that time delays and noise on the signals does not affect the stability of the system (e.g. introduce oscillations in power which also introduce oscillations in frequency). The DSO's proposed control achieved better results compared to the existing control in terms of voltage since it utilizes the ability of the WT's power electronics to absorb or produce reactive power in order to mitigate the voltage issues. The voltage profiles of $2 \mathrm{HV} / \mathrm{MV}$ substations in the HV side are presented in Figs. 1 and 2.

The resulting reactive power profiles of the 5 WTs of the second method are presented in Fig. 3.

Furthermore, the proposed control achieved higher penetration levels compared to the existing control as presented in Fig. 4.

This was mainly implemented by dispatching differently the WTs, utilizing more the WTs that have a lower rating if it is not secure to further increase the production of the WTs that a have higher rating. The profiles of the production of 2 WTs are presented in Figs. 5 and 6.

It is observed that the proposed control achieves lower production only in WT2 which has the highest nominal rating and results in higher production levels according 
to the existing DSO control. The proposed control reduces the power production of this WT in order to avoid a frequency transient that would result in a frequency nadir below $49.4 \mathrm{~Hz}$. The existing CHIL setup allows also to perform such transient in the RTDS in order to compare the two algorithms according to the frequency transients that could occur if any of them is under operation. In Fig. 7 the recorded transients in frequency for the disconnection of the largest producing WT were performed and recorded in different hours of the day proving the superiority of the proposed method.

To sum up, this test case allowed a second party (DSO) to test its control algorithms in an advanced testing setup of CHIL, not available at the DSO's premise, provided by a second party (ICCS). In this setup, the comparison was made in realistic conditions (noise, time delays) and for complex scenarios (WT outages) that could assist the DSO to evaluate and compare those 2 methods.

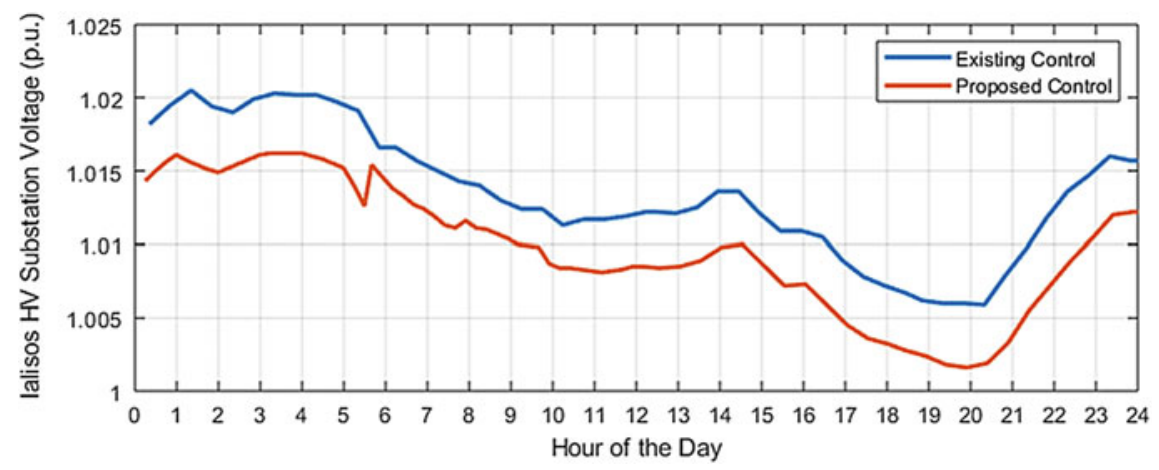

Fig. 1 Comparison of Ialisos HV profile

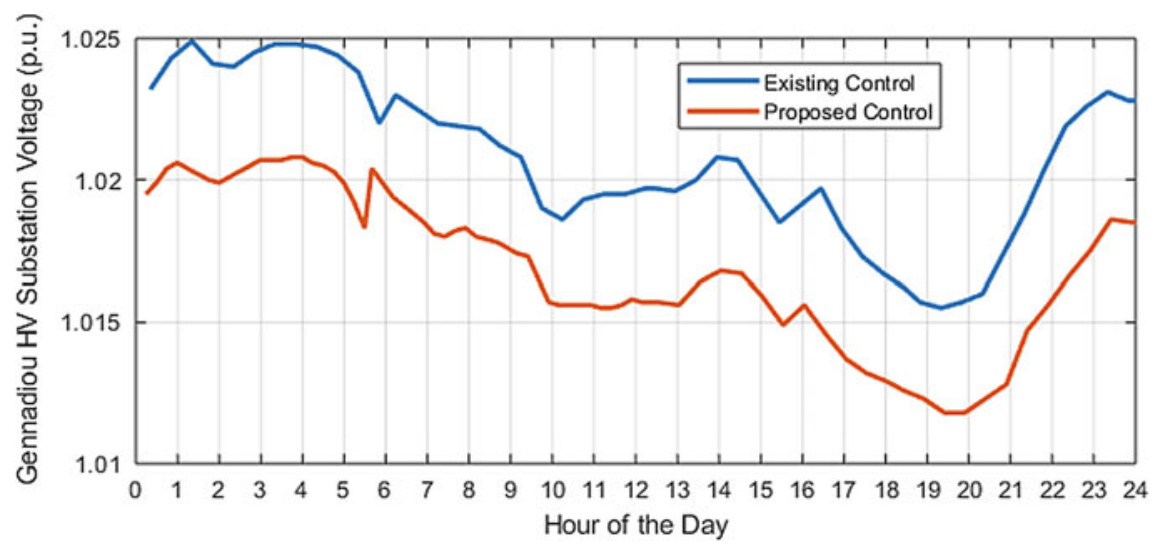

Fig. 2 Comparison of Gennadiou HV profile 


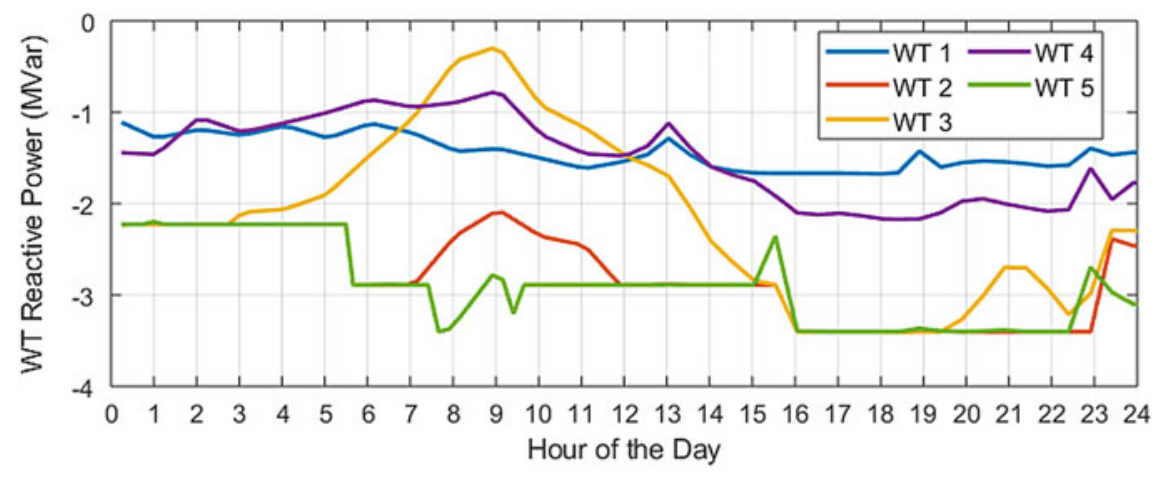

Fig. 3 Reactive Power Profiles throughout the day for the proposed HEDNO control

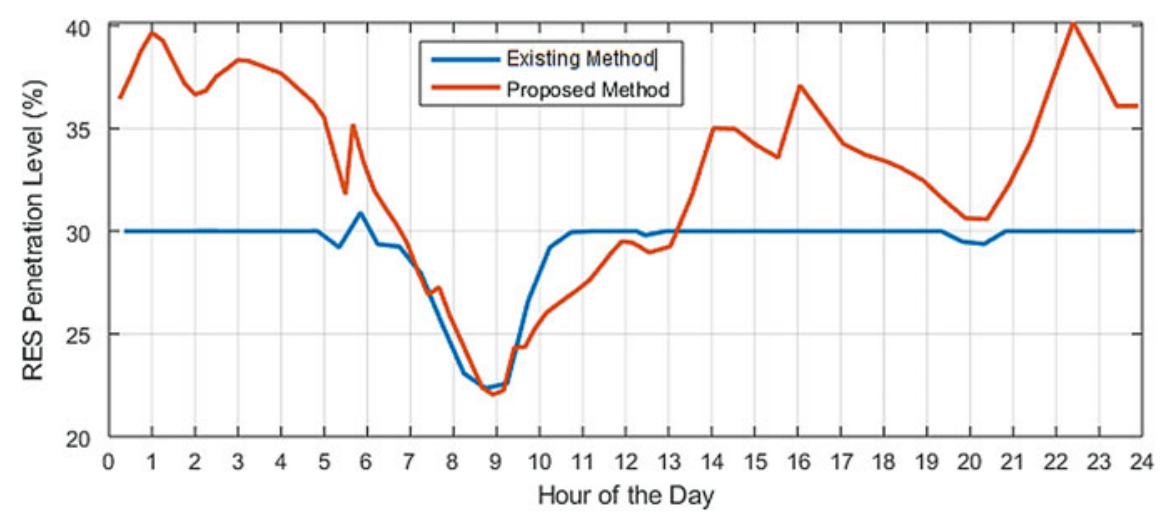

Fig. 4 Comparison of RES penetration levels for both controls

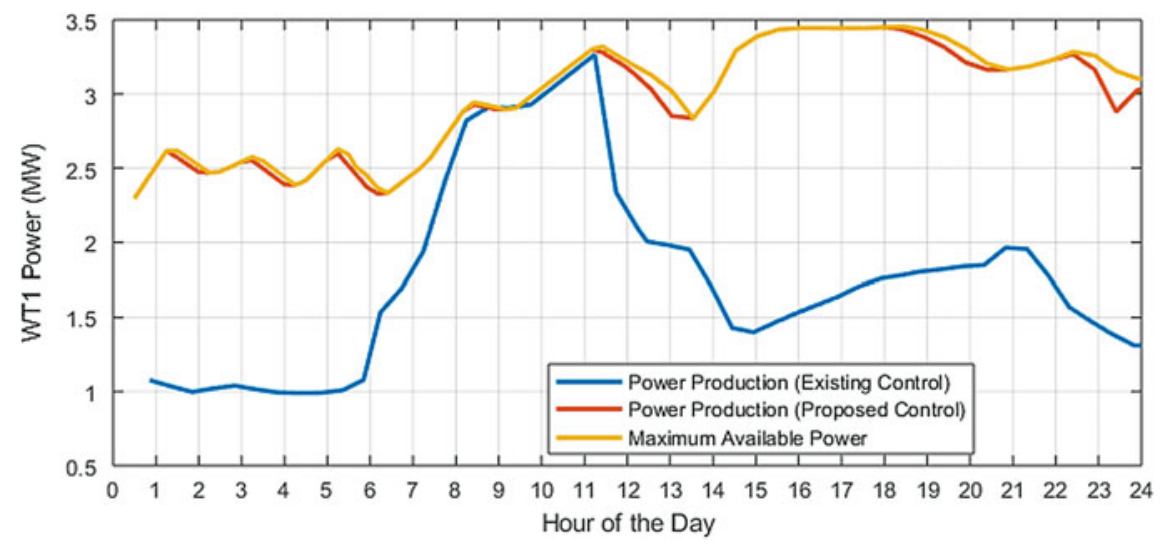

Fig. 5 Comparison of WT1 Power Production profiles 


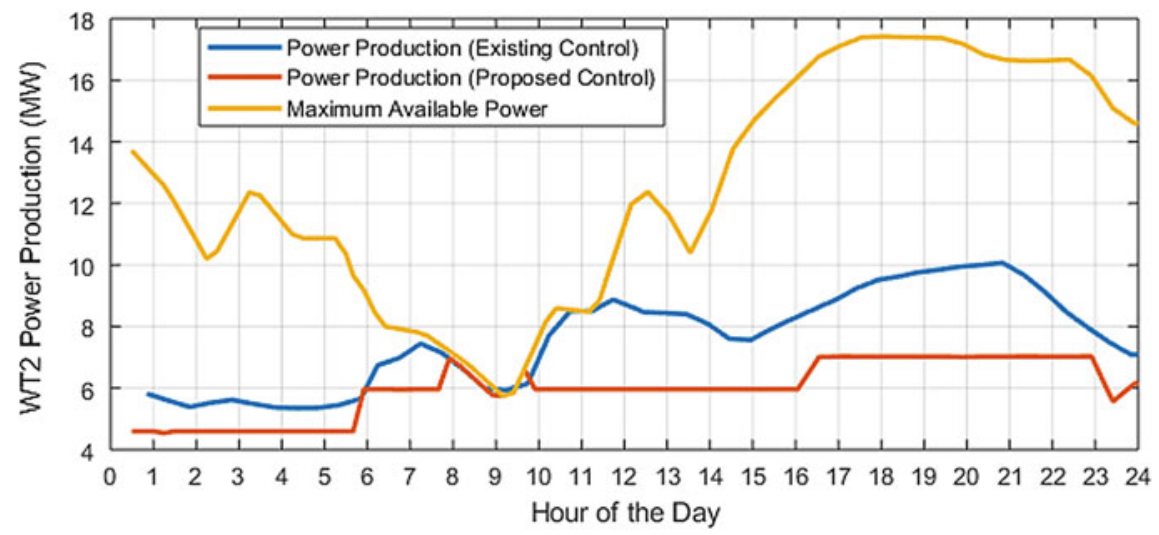

Fig. 6 Comparison of WT2 Power Production profiles

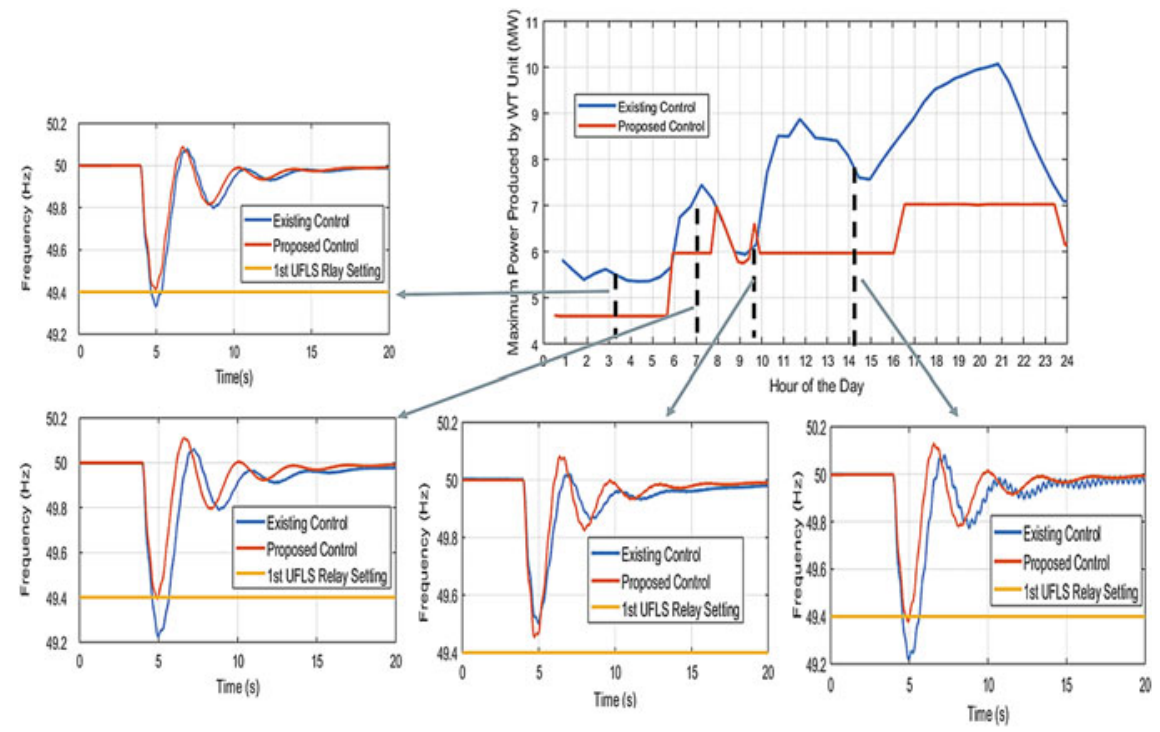

Fig. 7 Comparison of the frequency transients that could occur under each control algorithm operation

\subsection{Converter Controller Development}

This test case aims to demonstrate the "Testing Chain" approach. In order to demonstrate this approach, a characterization of a converter controller followed by a tuning was done. The test system includes: distribution LV grid, converter controller, PV, inverter. To characterize the converter controller a pure simulation test was implemented firstly, then after a tuning of the converter controller based on the simula- 


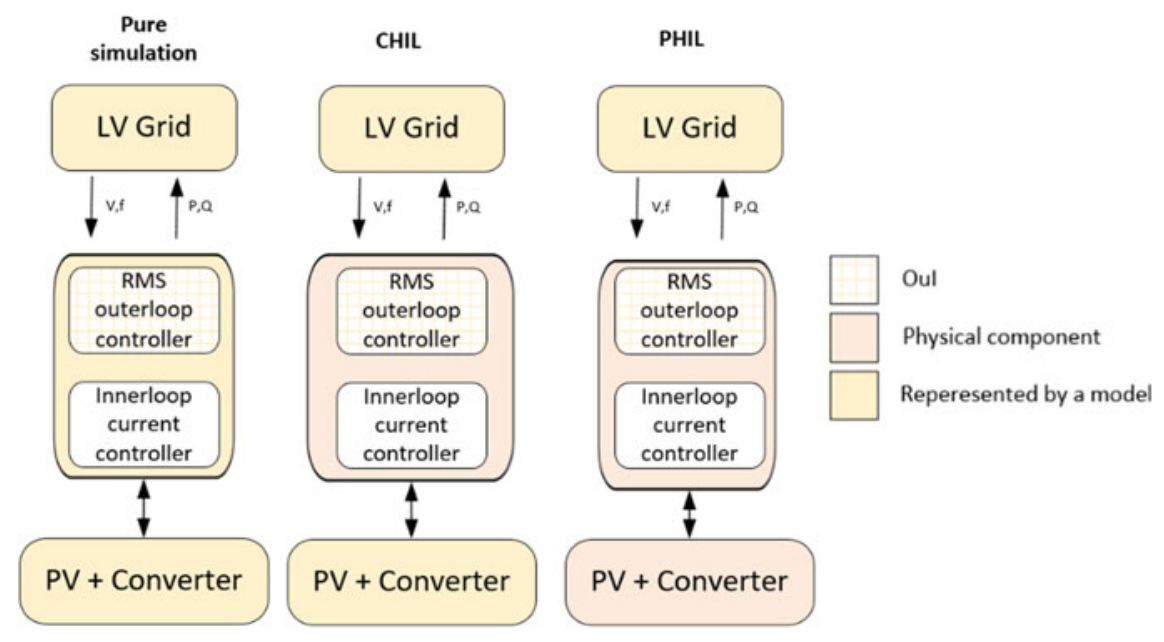

Fig. 8 System under test of the testing chain test

tion results, a CHIL and a PHIL test were performed to validate the improvements. Figure 8 shows the system under test taken into account in each step of the testing chain.

The selected LV test grid, considered as relevant enough for testing the converter controller, is based on the CIGRE LV network modified with DER that can be found in [1]. The object under test is a droop controller that is used to control the PV source in the grid. In order to compare the test results four KPIs have been defined and evaluated:

- Settling time (ST): Time elapsed from the application of an instantaneous step input to the time at which the amplifier output has entered and remained within an error band of $5 \%$.

- Overshoot (OS): $O S(\%)=\left(V_{\text {peak }}-V_{S S}\right) / V_{S S} \cdot 100$

- Time of peak (Tp): Time at which the peak value occurs.

- Damping factor (DF):

$$
\theta=\frac{\ln \left(\frac{O S}{100}\right)}{\sqrt{\pi+\ln ^{2}\left(\frac{O S}{100}\right)}}\left\{\begin{array}{lc}
\theta<1 & \text { Under damped } \\
\theta=1 & \text { Critically damped } \\
\theta>1 & \text { Over damped }
\end{array}\right.
$$

Following a description of the main results achieved with the testing chain.

\section{- Pure simulation test:}

To cover a wide spectrum of possible operating conditions, several experiment specifications have been defined, for analysing the converter response in case of generation and load variations. The experiment results are in Table 1. 
Table 1 Pure simulation results

\begin{tabular}{l|l|l|l|l|l}
\hline Test type & Action & ST [s] & OS [\%] & Tp [s] & DF \\
\hline Simulation & Step up PV output of 1 p.u. & 0.35 & 4.34 & 0.22 & 0.7 \\
\hline Simulation & $\begin{array}{l}\text { Step up of the grid load or } \\
\text { generation of 1 p.u. }\end{array}$ & 0.16 & 0.9 & 0.15 & 0.83 \\
\hline
\end{tabular}

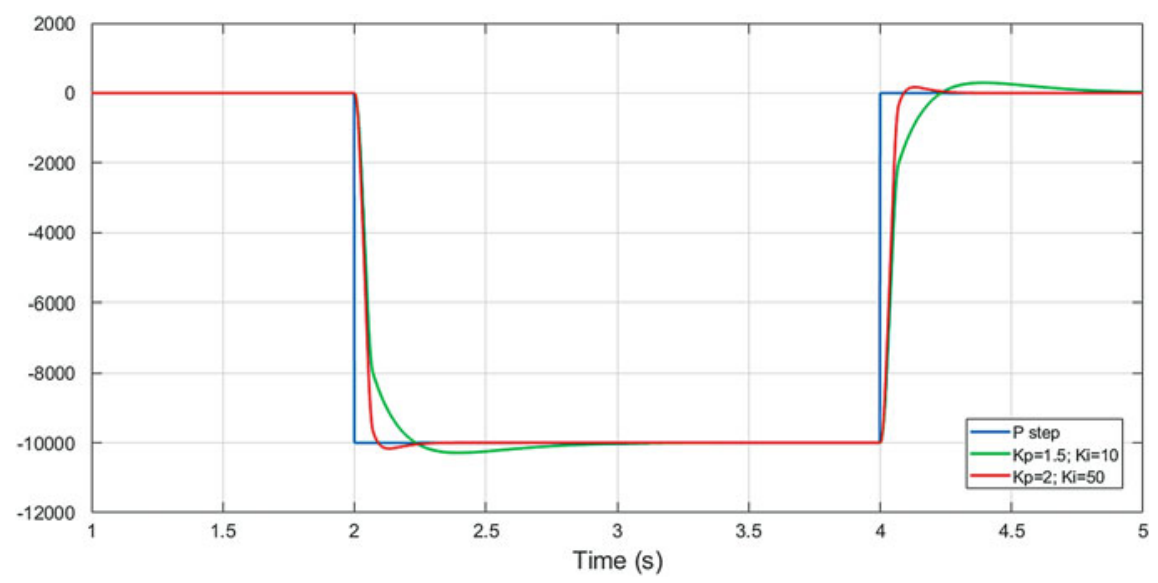

Fig. 9 Response of two $[\mathrm{Kp}, \mathrm{Ki}]$ sets of parameters

Based on these results the parameters of the converter controller were tuned further. Regarding the inner loop, a parametric analysis has been carried out to evaluate the impact of the PID constants in the system response when facing a step in the solar power generated. In any case, a wide range of values has been tested to find the better trade-off between the settling time and the overshoot. Two sets of [Kp, Ki] constants were selected. Figure 9 shows the new response of the converter controller compared to the old values of set [Kp, Ki].

In view of the results a final tuning of $\mathrm{Kp}=2$ and $\mathrm{Ki}=50$ has been eventually selected for the second round of tests.

Regarding the outer loop, potential improvements are linked to the adjustment of the measurement filters in the Id and Iq, and being more concrete, in the adjustment of the damping ratios (D). The damping ratio is a parameter linked to the quality of the filter in the way a higher damping ratio means a higher quality. Also, in this case several values of $\mathrm{D}$ have been evaluated in order to establish the best solution. The experiment's results show that the most suitable value for the D parameter of the Iq filter is 0.5 while the recommendation of the $\mathrm{D}$ value for the Id filter for the second round of tests is 0.85 . 
Table 2 CHIL results

\begin{tabular}{l|l|l|l|l|l}
\hline Test type & Action & ST [s] & OS [\%] & Tp [s] & DF \\
\hline CHIL & Step up PV output of 1 p.u. & 0.02 & 0.73 & 0.1 & 0.94 \\
\hline
\end{tabular}

Table 3 PHIL results

\begin{tabular}{l|l|l|l|l|l}
\hline Test type & Action & ST [s] & OS [\%] & Tp [s] & DF \\
\hline PHIL & $\begin{array}{l}\text { Step up PV output of 1 p.u. with } \\
\text { the original converter controller }\end{array}$ & 0.0084 & 16 & 0.0012 & 0.49 \\
\hline PHIL & $\begin{array}{l}\text { Step up PV output of 1 p.u. } \\
\text { with the improved converter } \\
\text { controller }\end{array}$ & 0.0062 & 22 & 0.0014 & 0.43 \\
\hline
\end{tabular}

\section{- CHIL test:}

Both sets of controller parameters which were discussed in the previous paragraph have been tested also with a CHIL setup. The results with the original converter controller are in Table 2.

With the CHIL experiments, the improved controller shows that the ST of the system is lower than the original controller, but it has a very oscillatory behaviour. For large steps in active power injection, such as the one required by this scenario, the controller became unstable. Therefore, it is expected that the second version of the controller will behave worse also in reality (in terms of oscillatory response) than the first version of the controller.

\section{- PHIL test:}

The last step of the testing chain is the PHIL experiment. In this case the converter controller was implemented on a real power converter. Similar to the CHIL experiment, also in this case the results show that the improved converter controller reduces the TS at the expense of the level of the OS (Table 3).

\section{Conclusions}

This chapter described the progress from general scenario thinking towards more detailed system configurations, use cases and test cases. Validation examples were presented to demonstrate the usage. The main objective was to access the relevancy of testing needs for smart grid system development. The work has started with the development of generic system configurations which provide a high-level context for ERIGrid testing. The work has proceeded to define focal use cases which cover the whole range of smart grid activities relevant to ERIGrid. Based on focal use case collection, most relevant testing scenarios for have been defined on an abstract level, 
outlining the most important areas of smart grid testing. The application examples demonstrate usage of the process within different circumstances. A first example demonstrates hardware/software integration across research infrastructures. In this case, CHIL and dynamic modelling were used as testing techniques. The second example focuses on testing chain approach, progressing from simulation studies to CHIL and PHIL tests.

\section{References}

1. Kotsampopoulos, P., Lagos, D., Hatziargyriou, N., Faruque, M.O., et al.: A benchmark system for hardware-in-the-loop testing of distributed energy resources. IEEE Power Energy Technol Syst J 5(3), 94-103 (2018)

2. Kulmala, A., Maki, K., Sandroni, C., Pala, D., et al.: D-JRA1.3 Use case implementation plan. Deliverable D7.3, ERIGrid Consortium (2017)

3. Maki, K., Blank, M., Heussen, K., Bondy, E., et al.: D-JRA1.1 ERIGrid scenario descriptions. Deliverable D7.2, ERIGrid Consortium (2016)

4. Rossi, M., Carlini, C., Pala, D., Sandroni, C., et al.: D-JRA1.2 Focal use case collection. Deliverable D7.2, ERIGrid Consortium (2016)

Open Access This chapter is licensed under the terms of the Creative Commons Attribution 4.0 International License (http://creativecommons.org/licenses/by/4.0/), which permits use, sharing, adaptation, distribution and reproduction in any medium or format, as long as you give appropriate credit to the original author(s) and the source, provide a link to the Creative Commons license and indicate if changes were made.

The images or other third party material in this chapter are included in the chapter's Creative Commons license, unless indicated otherwise in a credit line to the material. If material is not included in the chapter's Creative Commons license and your intended use is not permitted by statutory regulation or exceeds the permitted use, you will need to obtain permission directly from the copyright holder.

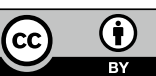

